\title{
Long-term Successful Antibiotic Therapy for Recurrent Aortic Graft Infection
}

\author{
Liana Dykman, Leore Cohen Mendel, Micha Rapoport \\ Department of Internal Medicine "C", Assaf Harofeh Medical Center, affiliated to Sackler Medical School, Tel Aviv University, Zerifin, Israel
}

Received: $12 / 05 / 2018$

Accepted: 15/05/2018

Published: 26/06/2018

How to cite this article: Dykman L, Cohen Mendel L, Rapoport M. Long-term successful antibiot therapy for recurrent aortic graft infection. EJCRIM 2018;5: doi:10.12890/2018_000913.

Conflicts of Interests: The Authors declare that there are no competing interests.

This article is licensed under a Commons Attribution Non-Commercial 4.0 License

\section{ABSTRACT}

Objective: To report a case of successful long-term conservative management of a patient with aortic graft infection due to multiple infectious agents.

Materials and methods: We describe the clinical case and present a review of relevant literature.

Results: An 82-year-old man presented with recurrent Escherichia coli bacteraemia. He was diagnosed with an endovascular aortic graft infection. As the patient declined surgery, conservative treatment with daily antibiotic therapy was instituted. We report good clinical results after almost 2 years of treatment and follow-up.

Conclusions: The preferred treatment of aortic graft infections is surgical. Conservative management is usually offered to poor surgical candidates and is associated with an unfavourable outcome. However, we report that selected patients may be successfully treated using prolonged antibiotic therapy.

\section{LEARNING POINTS}

- Endovascular graft infection is a serious, potentially life-threatening complication of aortic aneurism repair.

- Surgical management is the preferred therapeutic approach; information about conservative therapy options and their outcomes is limited.

- Long-term conservative management with ambulatory maintenance antibiotic therapy is an adequate treatment alternative and in selected patients can lead to a good clinical outcome.

\section{KEYWORDS}

Aortic graft infection, conservative management

\section{INTRODUCTION}

The use of endovascular grafts for the treatment of aortic aneurysms has increased significantly. Bacterial infections of these grafts, either in the perioperative period or later, are a feared complication with possibly fatal results. Although there are no clear guidelines for the management of these infections, antibiotic treatment is less accepted and the surgical approach (i.e. removal of the infected graft) is commonly adopted. We describe here a rare case of successful long-term antibiotic treatment of a recurrent graft infection due to multiple infectious agents. 


\section{CLINICAL CASE}

An 82-year-old man, whose past medical history includes smoking, hypertension, ischaemic heart disease, dyslipidaemia, benign prostatic hyperplasia, cholecystectomy and an abdominal aortic aneurysm treated by graft insertion 10 years previously, was admitted to our internal medicine department with recurrent episodes of general weakness, chills and high fever. Blood cultures were consistently positive for Escherichia coli. The patient was treated with IV antibiotics with resolution of fever and symptoms. Between the febrile episodes related to E. coli bacteraemia, the patient was asymptomatic and repeated blood cultures were negative. An extensive diagnostic and imaging work-up including renal US, CT urography, cystoscopy, colonoscopy, echocardiography and magnetic resonance cholangiopancreatography (MRCP) did not reveal the source of the recurrent infection.

However, CT angiography of the abdominal aorta demonstrated signs of aortitis in the area of the graft adjacent to the third part of the duodenum with a suspected aorto-enteric fistula. A PET-CT scan detected pathological uptake and wall thickening of the aortic graft consistent with infectious aortitis. The patient was offered surgical removal of the infected graft. He declined due to the substantial perioperative risk, so chronic daily maintenance treatment with intravenous ceftriaxone was administered via a permanent central catheter. More than 20 months have passed since diagnosis. During this period, the patient was admitted four more times with recurrent episodes of bacteraemia due to ESBL-positive E. coli and Candida albicans that were successfully treated with ertapenem and fluconazole, respectively. The patient continues to feel well under daily antibiotic therapy and to decline surgical treatment.

\section{DISCUSSION}

The incidence and prevalence of bacterial infections of endovascular grafts are significant, reaching $3 \%$ in different reviews ${ }^{[1]}$. These numbers are expected to grow in view of the preferential employment of these grafts to treat a variety of vascular pathologies ${ }^{[2]}$.

Graft infections are associated with a very high rate of mortality, which reaches $56.5 \%{ }^{[3]}$. The most common infectious organisms are Grampositive cocci, especially Staphylococcus aureus and Staphylococcus epidermidis, and to a much lesser extent, Gram-negative bacilli and E. coli infection, which is particularly rare ${ }^{[1]}$. The optimal management of prosthetic graft infection is unclear, but the common default approach is complete removal of the infected graft ${ }^{[3]}$. Some patients with very high perioperative risk may undergo full or partial graft preservation with instillation or irrigation of local antibiotics or continuous intravenous antibiotic treatment ${ }^{[1,4]}$, although there are no clear guidelines regarding conservative non-surgical treatment of these infections and only a handful of reports describe short-term conservative antibiotic treatment of endovascular graft infection $s^{[5]}$. As the use of endovascular stent grafting is becoming widespread, there is a growing need to deal with the complications of this procedure, namely infections. This is particularly true as patients undergoing this treatment are older and have a greater burden of chronic diseases ${ }^{[2]}$. Therefore, many patients are not candidates for the preferred surgical treatment or refuse it altogether, like our patient. Thus, the long-term clinical stability achieved in our patient with chronic ambulatory maintenance antibiotic therapy provides a practical and efficient therapeutic modality which may be adopted in a growing number of similar patients.

Future studies comparing surgical and conservative management of infected endovascular grafts are needed to determine which of these approaches is best in a particular patient.

\section{REFERENCES}

1. Saleem BR, Meerwaldt R, Tielliu IFJ, Verhoeven ELG, van den Dungen JJAM, Zeebregts CJ. Conservative treatment of vascular prosthetic graft infection is associated with high mortality. Am J Surg 2010;200:47-52.

2. Schwarze ML, Shen Y, Hemmerich J, Dale W. Age-related trends in utilization and outcome of open and endovascular repair for abdominal aortic aneurysm in the United States, 2001-2006. J Vasc Surg 2009;50:722-729.e2.

3. Garot M, Delannoy P-Y, Meybeck A, Sarraz-Bournet B, D'Elia P, D’Escrivan T, et al. Intra-abdominal aortic graft infection: prognostic factors associated with in-hospital mortality. BMC Infect Dis 2014;14:215.

4. Revest M, Camou F, Senneville E, Caillon J, Laurent F, Calvet B, et al. Medical treatment of prosthetic vascular graft infections: review of the literature and proposals of a Working Group. Int J Antimicrob Agents 2015;46:254-265.

5. Ladizinski B, Sankey C. Aortic graft infection. J Gen Intern Med 2014;29:1419-1420. 